\title{
Un instrument de démonstration au XVIIème siècle : le cadran sphère
}

Jean Pares

\section{(2) OpenEdition}

1 Journals

Édition électronique

URL : http://journals.openedition.org/trema/2434

DOI : 10.4000/trema.2434

ISSN : 2107-0997

\section{Éditeur}

Faculté d'Éducation de l'université de Montpellier

\section{Édition imprimée}

Date de publication : 1 mai 1992

Pagination : 69-74

ISSN : 1167-315X

\section{Référence électronique}

Jean Pares, «Un instrument de démonstration au XVIIème siècle : le cadran sphère », Tréma [En ligne], 1 | 1992, mis en ligne le 01 mai 1992, consulté le 01 mai 2019. URL : http://journals.openedition.org/ trema/2434 ; DOI : 10.4000/trema.2434

Ce document a été généré automatiquement le 1 mai 2019.

Trema 


\title{
Un instrument de démonstration au XVIIème siècle : le cadran sphère
}

\author{
Jean Pares
}

1 Expliquer le Monde et ses phénomènes en les reproduisant et en les modélisant à l'aide d'appareils, constitue l'une des bases de l'enseignement scientifique. Il semble actuellement difficile sinon impensable d'imaginer qu'un cours de sciences puisse se dérouler, du moins dans l'enseignement primaire et secondaire, sans l'appui d'instruments de démonstration et " d'expérience ».

2 Face aux magnifiques et abondants catalogues des maisons spécialisées, il existe des critiques, parfois violentes, portant sur la nature, la constitution et la finalité de ces instruments. Leur usage est malgré tout admis et pratiqué par le corps enseignant dans son ensemble et les objections contre cet usage ne semblent pas avoir ébranlé le consensus.

3 Loin des possibles controverses à leur sujet, il nous a semblé intéressant de rechercher un « ancêtre » de ces appareils didactiques et qui pourrait être encore utilisé de nos jours. Nous nous sommes pour cela tournés vers une période particulièrement riche en expériences pédagogiques et didactiques, le XVII ème siècle en l'occurrence.

4 A cette époque, parmi tous les systèmes mis en place par la Contre Réforme, l'enseignement fut l'un des plus efficaces et la principale Congrégation missionnaire en même temps qu'enseignante, celle des Jésuites, allait marquer pour longtemps les structures et les techniques pédagogiques.

5 Entre toutes les matières scientifiques, lorsqu'elles étaient enseignées dans leurs collèges, non seulement à leurs jeunes élèves mais aussi à des auditeurs extérieurs adultes, l'Astronomie et surtout l'initiation astronomique qui s'adresse à un large public, tenaient une place de choix.

6 Tenants souvent obligés du géocentrisme mais pourtant toujours zélés défenseurs de celui-ci sur ordre supérieur, les malheureux Jésuites firent découvrir le ciel et ses mystères à des générations d'élèves qui s'empressaient de les trahir en adoptant 
l'héliocentrisme. Sans doute leurs maitres éprouvaient-ils, intérieurement, quelque soulagement à les voir embrasser la doctrine copernicienne. Mais « perinde ac cadaver » ils continuaient à professer celle de Ptolémée et à baser leurs démonstrations sur cette dernière tout en exposant, en la réputant fausse et contraire aux Saintes Ecritures, celle qui valut tant d'ennuis à Galilée.

7 Ils ont, dans la plupart des cas connus, fondé cet enseignement sur l'étude et l'utilisation des instruments astronomiques d'observation et de calcul. Lorsqu'on sait le rôle prépondérant qu'a joué au XVII ${ }^{\text {ème }}$ siècle l'instrumentation dans la construction et l'affermissement de la nouvelle astronomie, le choix des Jésuites apparait fort judicieux. Sans pourtant diminuer leur mérite, notons que l'usage d'instruments didactiques pour l'enseignement de l'astronomie, a été courant au Moyen-Age et sans doute dans l'Antiquité hellénique. Ces instruments étaient alors en même temps appareils d'enseignement et de calcul mais non d'observation, bien qu'ils aient permis des visées grossières, comme c'est le cas, par exemple, pour les astrolabes planisphériques.

8 Ainsi tout un ensemble d'instruments anciens et modernes a été utilisé par les Jésuites et ce, dès le XVI ème siècle. Dans cet ensemble, nous retiendrons en particulier un appareil modeste, récent à l'époque et plein d'opportunités sur le plan didactique. Il s'agit du cadran sphère, constitué tout simplement par un globe terrestre et dont la première description, d'ailleurs entachée d'erreurs, figure dans le «De solaribus horologiis» (1531-32) d'Oronce Finé qui ne semble pas avoir compris le pourquoi de l'appareil.

Figure 1 : Globe Tellure in « Récréations scientifiques » G. Tissandier Masson éditeur 1883

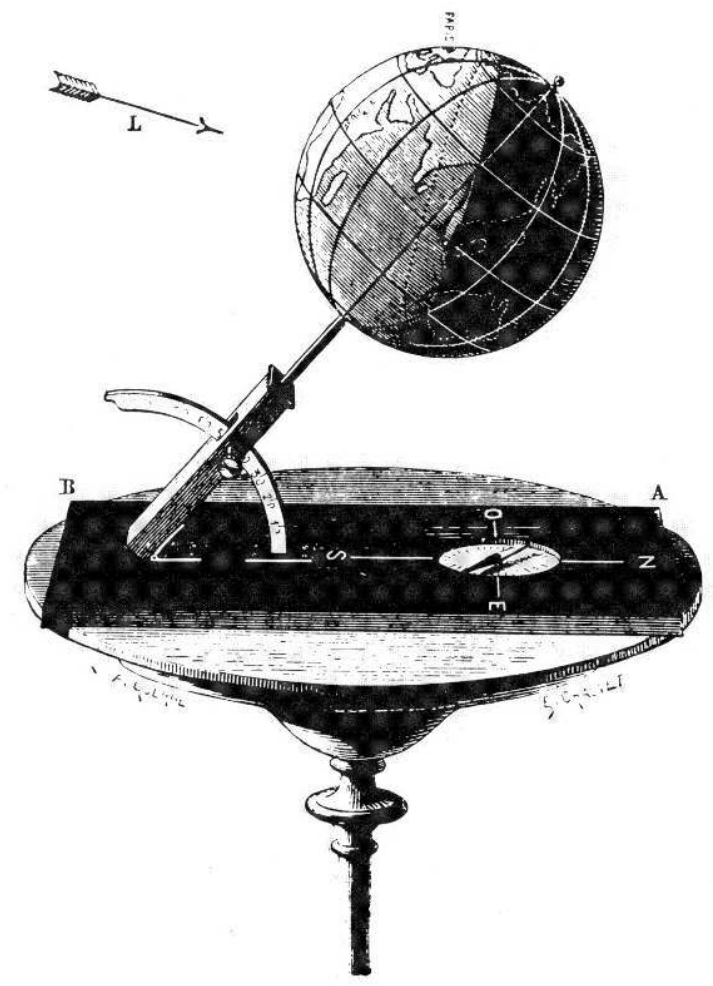

9 Ce cadran possède la particularité et l'avantage de reproduire exactement et en temps réel le phénomène d'éclairement de la Terre par le Soleil.

Alors que l'idée de sphéricité de notre planète a été proposée par les pythagoriciens dès le IV ${ }^{\text {ème }}$ siècle avant notre ère, certes pour des raisons non scientifiques mais esthétiques, ce 
qui n'en avait à l'époque que plus de force, il est curieux de constater que ne soit pas née alors, en même temps ou par la suite, l'idée de représenter l'effet des rayons solaires sur la Terre grâce à un appareil où le Soleil jouerait son propre rôle tandis que la Terre serait matérialisée par une petite sphère. L'Ecole d'Alexandrie, pourtant grande productrice de mécanismes complexes représentant les mouvements célestes et de cadrans et quadrants solaires savants, ne semble pas, dans l'état de nos connaissances, avoir exploité cette idée simple et facile à concrétiser.

11 Le cadran sphère est, en effet, constitué par une sphère munie des mêmes lignes et points remarquables qu'un globe terrestre. Il est évident que la géographie «scientifique » aux époques précitées n'avait pas encore transposé les cercles de la sphère céleste à la sphère terrestre, même si le diaphragme et les climats annonçaient longitudes et latitudes terrestres. D'autre part, l'usage des heures temporaires ne liait pas les lignes d'heures et les longitudes comme c'est le cas pour nos heures (égales ou équinoxiales). Aussi tout ceci pourrait bien être la raison de la non conception du cadran sphère dans l'Antiquité.

Pour utiliser l'appareil, il faut placer sa ligne des pôles parallèlement à la ligne des pôles terrestres (ou célestes). Le point de la sphère correspondant au lieu où se trouve le cadran doit se trouver au sommet de cette sphère.

Figure 2 : Le positionnement d'un cadran sphère. NAESE' est le méridien du lieu A du cadran. NS est orienté parallèlement à l'axe du Monde. NAESE' se trouve dans le plan du méridien local. Par construction A est au sommet du globe.

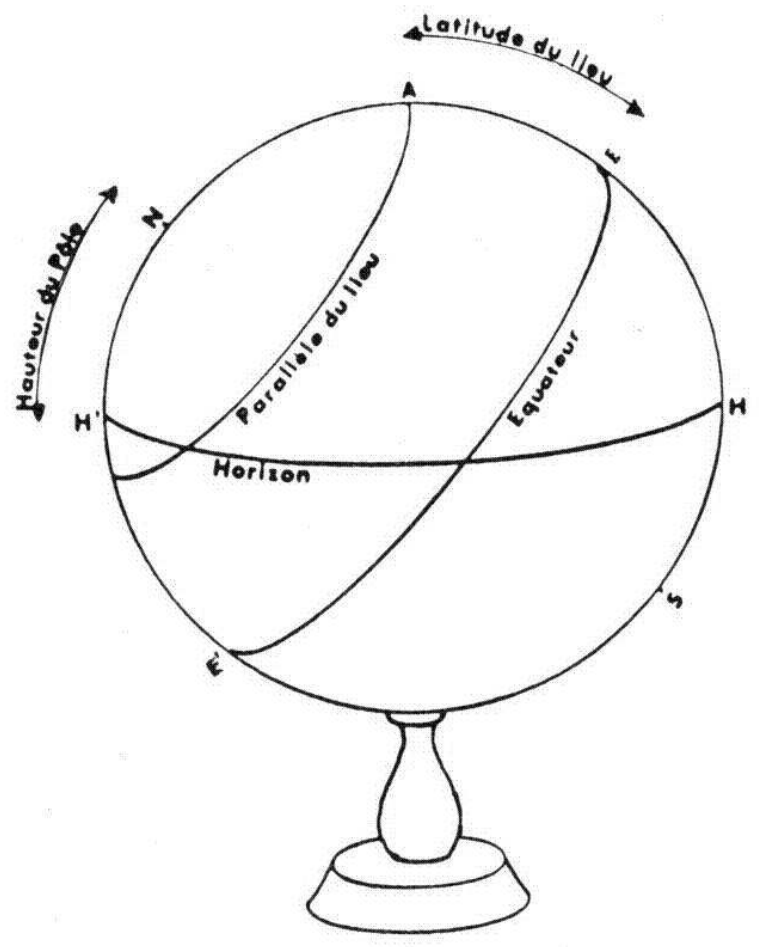

Les lignes d'heures sont alors constituées par des méridiens distants de $15^{\circ}$ entre eux, le premier de ceux-ci correspond à la ligne de oh (ou $12 \mathrm{~h}$ ou même $6 \mathrm{~h}$ suivant le type de cadran) et passe par le point représentant le lieu du cadran et doit se trouver placée dans le $1^{\text {er }}$ vertical du lieu d'observation. Le cercle de latitude passant par ce point constitue une ligne d'heure particulière pour indiquer les heures italiques, comptées à partir du coucher du soleil et les babyloniques comptées à partir de son lever. Le tracé sur le globe 
des terres, mers et pays permet de situer à un instant donné la position de l'ombre et de la lumière donnée par le soleil et d'obtenir ainsi une série d'indications intéressantes.

L'appareil, d'abord très simple - ainsi à ses débuts seul l'équateur était gradué en heures par ses intersections avec les méridiens horaires -va se perfectionner, se « sophistiquer » au cours du XVII ème siècle en particulier grâce à deux Jésuites: Athanase Kircher et Gaston Pardiès, le premier dans son « Ars Magna Lucis et Umbrae » où il développe toutes les possibilités de la sphère du point de vue gnomonique avec le secours de la Physique, le second dans un traité " Deux machines à faire les quadrans » où, nous dit-il, son appareil «marque les diverses heures, les signes du zodiaque, les degrés de hauteur. On y connait à chaque moment quels sont les endroits de la Terre qui sont éclairés du soleil, et quels sont ceux qui ont de longues nuits; on y distingue vers les pôles tous les endroits qui ont une nuit perpétuelle, ou qui voyent le Soleil sans interruption; les heures italiques et les babyloniques, la grandeur des crépuscules, la durée des jours et des nuits, les lignes ascendans et les descendans, les Maisons célestes et tout le reste qui feraient un épouvantable embarras dans les quadrans ordinaires se voient ici sans aucune confusion ». Ajoutons, entre autres, la possibilité de connaître combien d'heures avant ou après six heures, le Soleil se lève à une date donnée. Tel quel, ce cadran lie très concrètement des phénomènes physiques naturels à la notion de temps.

Figure 3 :
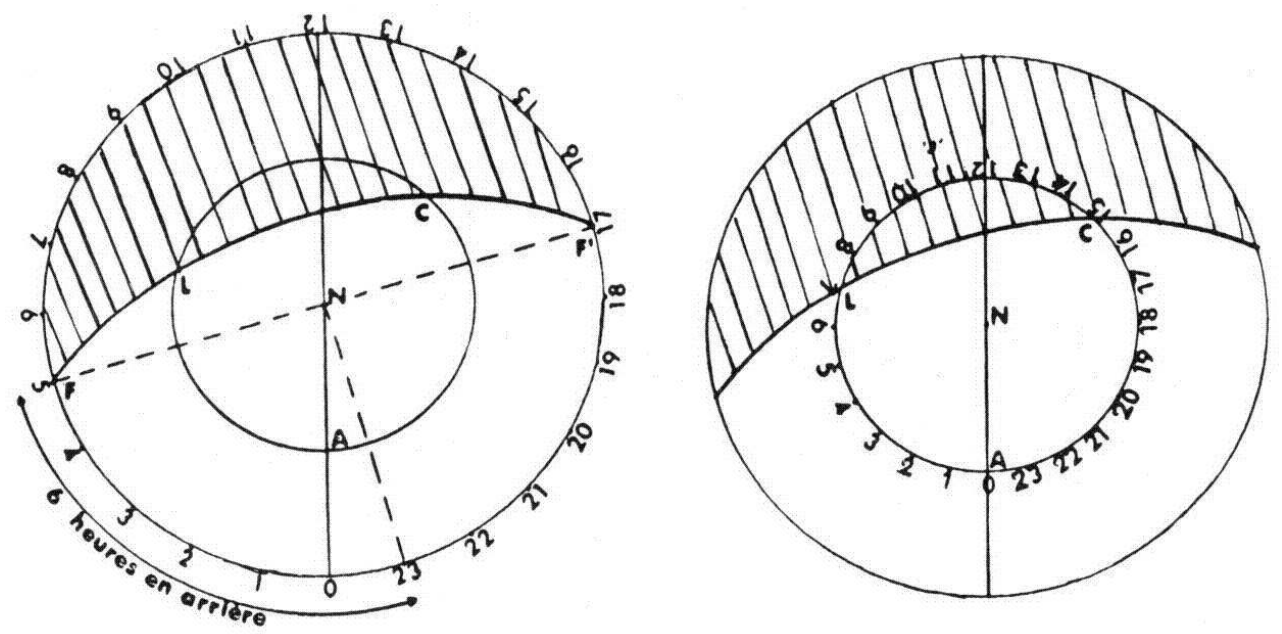

Cadran sphère (vue de dessus). La partie éclairée est vers le bas de la figure, au dessous du cercle de séparation FLCF'. Ce cercle passe par les points de 5 h et $17 \mathrm{~b}$ sur l'Equateur. L'heure astronomique est alors $23 \mathrm{~h}(17+6=23$ ou 5-6=-7, c'est-à-dire une heure avant midi) ou encore il est $17 \mathrm{~h}$ vulgaire (1/2(5+77)-17). Même cadran (même instant). Le cercle de séparation coupe le cercle de latitude du lieu A du cadran en $7 \mathrm{~h}$ et $75 \mathrm{~h}$ : il est $7 \mathrm{~h}$ babylonique et $75 \mathrm{~h}$ italique; soit 7 heures écoulées depuis le lever du Soleil (passage du point L en A) ou 75 heures écoulées depuis le coucher précédent du Soleil (passage de C en A). Le nombre d'heures dans la zone d'ombre entre $L$ et $C$ indique la durée de la nuit : 8 heures. Le nombre entre $C$ et $L$, dans la zone éclairée, indique la durée du jour: 16 heures.

En conclusion, le cadran sphère, somme de connaissances astronomiques et géographiques élémentaires, certes, mais néammoins souvent difficiles à faire comprendre ou même admettre, constitue un moyen simple et tangible pour enseigner les phénomènes qu'il reproduit naturellement. Ne nécessitant, après son positionnement aucune autre manipulation, instrument passif en quelque sorte, il reproduit mieux le réel, puisqu'il y participe, que les appareils de démonstration généralement proposés aux élèves. 
16 Malgré ces possibilités, en dehors de son utilisation par les Jésuites, nous n'avons trouvé aucune mention de l'emploi des cadrans sphère dans l'enseignement moderne. Seul, parmi tous les ouvrages de vulgarisation du XIX ${ }^{\text {ème }}$ siècle, en 1883, les "récréations scientifiques » de Gaston Tissandier, rédacteur du journal «La Nature», donnaient la description et l'usage d'un "globe tellure » qui n'était rien d'autre qu'un cadran sphère modernisé, mais elles n'indiquaient que quelques possibilités didactiques de l'appareil.

Il ne semble pas que l'appareil, même sous cette dernière forme, ait tenté les fabricants de matériel d'enseignement ou qu'ils aient indiqué la possible utilisation des globes terrestres comme cadrans sphère, ce qui aurait ajouté un "plus » à leurs arguments de vente et peut-être plus de facilités à l'enseignement élémentaire des mouvements de la Terre et de leurs conséquences ${ }^{1}$.

BIBLIOGRAPHIE

\section{1) Ouvrages traitant des cadrans sphère.}

Gaston Ignace PARDIES : Description et explication des deux machines propres à faire les cadrans avec une grande facilité. Paris 1673.

Athanase KIRCHER : Ars magna lucis et umbrae. Rome 1645.

Pierre de SAINTE MARIE MAGDELAINE : Traité d'Horlogiographie. Paris 1641.

Nicolas BION : Traité de la construction et des usages des instruments mathématiques. Paris 1709. Jean PARES : La Gnomonique de Desargues à Pardiès. Cahier d'Histoire et de Philosophie des sciences n¹7. Belin 1988.

\section{2) Ouvrages contenant des représentations de cadrans sphère}

Les cadrans solaires de Max ELSKAMP. Musée de la vie Wallonne. Liège 1966

Henri MICHEL : Les instruments des sciences dans l'Art et l'Histoire. Société Française du Livre. Paris 1966 ou Albert de Visscher Editeur 1980 (Belgique)

\section{3) Les ouvrages de gnomonique ne citent que rarement et très succinctement les cadrans sphère}

(ceux-ci ne tenant qu'une place très modeste dans l'Art des cadrans). Parmi eux, le meilleur actuellement est certainement celui de : René R-J. ROHR : Les cadrans solaires. Edition Oberlin. Strasbourg 1986. 


\section{NOTES}

1. Les anciens, jusqu'aux modernes, divisaient les périodes diurnes et nocturnes, chacune en douze parties égales. Ces parties étaient donc de durées différentes pour le jour et la nuit, sauf aux équinoxes.

\section{AUTEUR}

\section{JEAN PARES}

Maître de conférences, université de Montpellier II, USTL 